\title{
ALTERAÇÕES COMPORTAMENTAIS E NEUROQUÍMICAS DURANTE A FASE AGUDA DO PROCESSO CONVULSIVO INDUZIDO POR PILOCARPINA
}

\author{
Behavioural and neurochemical alterations during the acute phase of \\ convulsive process induced by pilocarpine
}

\author{
Rivelilson Mendes de Freitas \\ Professor Adjunto do Setor de Farmacologia da Universidade Federal do Piauí - UFPI. \\ Rua Cícero Eduardo, s/n, Junco, Picos, 64600-000, Piauí.
}

*Autor para correspondência e-mail: rivelilson@ufpi.br

Recebido em 19/08/2008 - Aceito em 17/11/2008

\begin{abstract}
RESUMO: Estudos comportamentais e neuroquímicos em diferentes áreas cerebrais foram realizados em animais adultos (2 meses de idade) que apresentaram convulsão e estado epiléptico (EP), através da administração de pilocarpina (400 mg/kg, s.c., P400), com a finalidade de investigar as atividades das enzimas superóxido dismutase (SOD) e catalase (CAT) durante a fase aguda (1 e $24 \mathrm{~h}$ ) do processo convulsivo. Os estudos comportamentais em animais adultos mostraram que a administração de P400 produziu sinais colinérgicos periféricos e movimentos estereotipados em todos os animais nos dois períodos de observação. Os animais observados por $1 \mathrm{~h}$ apresentaram parâmetros comportamentais semelhantes ao grupo de $24 \mathrm{~h}$, só que 0 índice de desenvolvimento de convulsões, EP, foi um pouco menor, e não houve nenhuma morte. Os estudos neuroquímicos revelaram que a atividade da SOD aumentou durante a primeira hora em todas a áreas. Por sua vez, no período de $24 \mathrm{~h}$ aumentou apenas no córtex frontal. Na atividade da catalase em ambos os períodos e nas áreas estudadas verificou-se um aumento significativo. Nossos resultados indicam que as convulsões induzidas por pilocarpina apresentam alterações comportamentais características da atividade epiléptica e pode haver também um incremento considerável nas atividades das defesas antioxidantes estudadas, na tentativa de proteger o animal contra o dano neuronal produzido pelo estresse oxidativo observado nas estruturas cerebrais estudadas. As alterações comportamentais e nas atividades da SOD e CAT observada sugere uma extensa participação do hipocampo, do corpo estriado e do córtex frontal de ratos adultos na instalação, propagação e/ou manutenção do processo convulsivo induzido por pilocarpina.
\end{abstract}

PALAVRAS CHAVES: Convulsões, Estado epiléptico, Pilocarpina, Superóxido Dismutase, Catalase.

\begin{abstract}
Neurochemical and behavioral studies in different brain areas were realized in adult rats (2 monthsold) that presented seizures and status epilepticus (SE), induced by pilocarpine $(400 \mathrm{mg} / \mathrm{kg}, \mathrm{sc}, \mathrm{P} 400)$, with the aim of investigate the activities of enzymes superoxide dismutase (SOD) and catalase (CAT) during the acute phase (1 and 24) of convulsive process. The behavioral studies in adult animals showed that the administration of P400 produced signs cholinergic peripherals and stereotyped movements in all animals in the two periods of observation. The animals observed by $1 \mathrm{~h}$ showed similar behaviour to the group of 24 , only that the rate of development of seizures, SE, was slightly reduced, and there was no death. The neurochemical studies revealed that the SOD activity increased during the first hour in all the areas. In the period 24 hours increased only in frontal cortex. In catalase activity in both periods and in areas studied there was a significant increase. Our results indicate that the seizures induced by pilocarpine have behavioural change characteristics of epileptic activity and there may also be a considerable increase in the activities of antioxidant defenses studied, in an attempt to protect the animal against neuronal damage produced by oxidative stress observed in brain structures studied. The behavioural change and the activities of SOD and CAT observed suggests an extensive participation of the hippocampus, striatum and the frontal cortex of adult rats at the installation, propagation and/or maintenance of convulsive process induced by pilocarpine.
\end{abstract}

KEYWORDS: Seizures, Status epilepticus, Pilocarpine, Superoxide Dismutase, Catalase. 
Freitas, R. M. et al./Revista Eletrônica de Farmácia Vol 5(3), 60 - 70, 2008.

\section{INTRODUÇÃO}

A epilepsia do lobo temporal (ELT) é uma doença crônica que pode interferir em inúmeros aspectos sociais e psicológicos do paciente e ainda está freqüentemente associada a um estímulo inicial precipitante, tais como: estado epiléptico, trauma e convulsões febris prolongadas (DODRILL, 2004). Sendo, um dos distúrbios neurológicos mais comuns, apresenta uma taxa de prevalência de 5\% (DE LORENZO et al., 2001). Aproximadamente, 30 a $50 \%$ dos pacientes epilépticos são sintomáticos, e esta condição pode ser adquirida através de um stress ambiental (DE LORENZO et al., 2000).

A ELT pode ser definida como uma afecção crônica de etiologia diversa, caracterizada por crises repetidas, devido a uma descarga excessiva dos neurônios cerebrais em diferentes estruturas e associada eventualmente com diversas manifestações clínicas e paraclínicas, podendo ocorrer também quando há um aumento do nível basal de excitação do Sistema Nervoso Central (SNC), superior ao limiar da convulsão (DE LORENZO et al., 2001).

As crises convulsivas podem ser associadas às diversas mudanças bioquímicas em algumas áreas cerebrais afetando vários neurotransmissores (MICHOTTE et al., 2000), com também induz alterações no metabolismo dos carboidratos, nos sistemas de segundos mensageiros e na expressão gênica, que poderiam estar envolvidos na fisiopatologia responsável pelas alterações ao longo do tempo nos neurônios (SIMONIC et al., 2000).

Os modelos de epilepsia do lobo temporal semelhante a dos humanos podem ser utilizados para estudar as mudanças neuroquímicas relatadas durante o desenvolvimento e na propagação e/ou manutenção das convulsões, (MARINHO et al., 1997), e também pode ser útil para caracterizar prontamente os mecanismos envolvidos na epilepsia.

A fisiopatologia da epilepsia não estar completamente definida. O modelo de convulsão induzido por pilocarpina em animais é bastante utilizado para estudar a fisiopatologia do processo convulsivo, uma vez que, reproduz as alterações comportamentais e eletroencefalográficas que são semelhantes à epilepsia do lobo temporal de humanos (BEM-ARI et al., 1981). Este modelo pode também ser utilizado para estudar o envolvimento dos sistemas de neurotransmissores como moduladores da epileptogênese, como também permite observar as alterações comportamentais, histopatológicas, e outros parâmetros neuroquímicos relacionados com a atividade epiléptica (COSTA-LOTUFO et al., 2002).

No modelo de epilepsia induzido por alta dose de pilocarpina, pode-se observar perda neuronal de algumas áreas cerebrais, a saber: hipocampo, corpo estriado, amígdala, córtex piriforme, córtex entorrinal, tálamo e substância negra, sugerindo o envolvimento dessas diferentes áreas durante o estabelecimento do processo epiléptico (BORELLI \& BOZZI, 2002).

Entre as áreas em que ocorre dano neuronal, o hipocampo, o corpo estriado e o córtex fronto-parietal, além de serem as áreas mais acometidas, podem estar relacionadas de forma importante com os mecanismos de instalação, da propagação e/ou manutenção (epileptogênese) das convulsões límbicas (MARINHO et al., 1998). Em geral, as convulsões induzidas por pilocarpina parecem depender da ativação do receptor muscarínico, da alteração da atividade enzimática de alguns sistemas, entre eles os sistemas de defesa antioxidante, do metabolismo dos fosfoinositídios (MARINHO et al., 1998), como também da participação de outros sistemas de neurotransmissão noradrenérgico (CAVALHEIRO et al., 1994), dopaminérgico, serotonérgico (CAVALHEIRO et al., 1995), GABAérgico e glutamatérgico.

Dentre as inúmeras mudanças neuroquímicas vistas durante a fase aguda do processo convulsivo tem sido verificada expressivas alterações na produção de lipídio peróxidos, no conteúdo do nitrito e nitrato, na concentração da glutationa reduzida (GSH), nas atividades enzimáticas da superóxido dismutase (SOD) e catalase (CAT). De um modo geral, os radicais livres têm sido implicados na toxicidade de numerosos agentes químicos e na patogênese de muitas doenças, tais como: doenças inflamatórias e patologias do SNC (Parkinson, Alzheimer, demência e epilepsia). A lista dessas doenças é cada vez maior e isso se deve, pelo menos em parte, ao fato de que essas moléculas reativas podem produzir a maioria das alterações teciduais, que têm sido identificadas em uma grande variedade de processos danosos. Muitas dessas alterações, porém, podem ser uma conseqüência e não a causa do dano (KEHRER, 1993).

O organismo humano está exposto a vários radicais livres e "espécies reativas derivadas do oxigênio" (EROs) geradas por radiações ionizantes, agentes tóxicos, poluentes ambientais, nos processos patológicos, etc. Os neurônios cerebrais são também capazes de produzir EROs, tais como: o peróxido de hidrogênio e os radicais superóxido, hidroxila e óxido nítrico. Visto que participam de reações essenciais para o organismo, as EROs são constantemente formadas e podem ser prejudiciais, quando sua produção ultrapassa o controle exercido por sistemas antioxidantes, endógenos de defesa (SIES, 1991).

Devido ao seu grande potencial citotóxico dos radicais livres e das EROs produzidas no organismo sob rigoroso controle dos sistemas de defesa que incluem enzimas e outros antioxidantes (HALLIWELL \& GUTTERIDGE, 1992), seria importante verificar a ação da enzima SOD presentes na mitocôndria e citossol que converte o radical superóxido em peróxido de hidrogênio (HALLIWELL E GUTTERIDGE, 1992), como também a 
Freitas, R. M. et al./Revista Eletrônica de Farmácia Vol 5(3), 60 - 70, 2008.

outra enzima antioxidante endógena CAT que catalisa a redução de peróxido de hidrogênio em água e oxigênio. Ambas podem ser encontradas nas diferentes áreas cerebrais desempenhando importante papel neuroprotetor.

Com base na literatura revisada, o estresse oxidativo (EO), é uma das condições de dano celular mais estudada atualmente, tem sido implicado em muitos processos patológicos. Dentre eles, a epilepsia, inflamação, artrite reumatóide, câncer, aterosclerose e dano de reperfusão, as quais têm bases teórica e experimental que sustentam de forma mais consistente, um papel causal ou contributivo para o $\mathrm{EO}$. O cérebro é mais vulnerável que outros tecidos ao EO, por que contém uma grande quantidade de lipídios e metais oxidáveis, e têm em relação aos outros tecidos menos mecanismos antioxidantes (WALZ et al., 2000).

O EO definido como a excessiva produção de radicais livres pode alterar dramaticamente a função celular, e modificar a liberação e/ou síntese de outros compostos que podem estar relacionados com a morte neuronal induzida pela convulsão e tem sido implicado em uma variedade de condições neurológicas agudas e crônicas, incluindo a epilepsia (FRANTSEVA et al., 2000).

Foi sugerido que o dano neuronal observado após o EP induzido por pilocarpina pode ser resultante da produção exagerada de radicais livres, tais como: peróxido de hidrogênio, radical hidroxila e superóxido (BOVERIS et al., 1986). Apesar, dessas evidências o envolvimento das EROs durante o período agudo das convulsões induzidas pela pilocarpina precisa ser melhor esclarecido. Como também é necessário estudar as possíveis alterações nos sistemas antioxidantes responsáveis pela remoção das EROs nas diferentes estruturas cerebrais relacionadas com a atividade epiléptica.

Visto que o mecanismo através do qual a pilocarpina produz convulsões e EP ainda não está totalmente esclarecido e estas convulsões são uma importante neuropatologia de interesse para a saúde pública, devido à sua alta prevalência, os objetivos deste trabalho foi investigar as alterações comportamentais observadas durante a fase aguda ( 1 e $24 \mathrm{~h}$ ) do processo convulsivo induzido por pilocarpina em alta dose $(400 \mathrm{mg} / \mathrm{kg})$. Como também verificar a atividade das enzimas antioxidantes: superóxido dismutase e catalase em diferentes áreas cerebrais envolvidas no estabelecimento do foco epiléptico (hipocampo, córtex frontal e corpo estriado) de ratos Wistar adultos.

\section{MATERIAL E MÉTODOS}

\section{Animais}

Foram utilizadas ratos Wistar machos, adultos (2 meses de idade, 250 - 280g), provenientes do Biotério Central do Departamento de Fisiologia e Farmacologia, da Faculdade de Medicina da Universidade Federal do Ceará (UFC). Durante todos os experimentos, os animais foram mantidos em gaiolas de acrílico de $30 \times 30 \times 30$ $\mathrm{cm}^{3}$ onde foram observados por 1 e $24 \mathrm{~h}$ com no máximo 6 animais, em condições controladas (temperatura ambiente de $24-25^{\circ} \mathrm{C}$ e umidade de 50 a $60 \%$ ), com ciclo claro / escuro alternado de 12 horas (07:00 am a 07:00 pm), recebendo ração padrão tipo Purina e água ad libitum. Os experimentos foram realizados de acordo com o guia de cuidados e usos de animais de laboratório do Departamento de Saúde e Serviços Humanos dos Estados Unidos da América (EUA).

\section{Tratamentos}

Os animais foram tratados com pilocarpina (400 mg/kg, sc, $\mathrm{n}=40, \mathrm{P} 400)$ e observados por $1 \mathrm{~h}$ (P400 1h) e 24 horas (P400 24h) após a administração. Os animais controles foram tratados com solução salina ( $\mathrm{NaCl}, 0,9 \%$, $\mathrm{n}=40$, Controle). Após $1 \mathrm{~h}$ e 24 horas da administração de pilocarpina, os animais que apresentaram convulsões, estado epiléptico e sobreviveram ao tratamento foram sacrificados sem anestesia por decapitação em guilhotina (Havard, USA), e em seguida, seus cérebros foram removidos. O hipocampo, o córtex frontal e o corpo estriado foram dissecados sobre gelo e armazenados em papel alumínio devidamente identificado, pesado e conservado a $-80^{\circ} \mathrm{C}$ para uso posterior. As dosagens neuroquímicas foram realizadas após $24 \mathrm{~h}$ da dissecação.

\section{Estudo comportamental}

Os animais tratados e controles foram divididos em gaiolas contendo no máximo seis animais e colocados em ambiente reservado, sendo feita à observação direta. Todos os grupos experimentais foram observados após a administração da pilocarpina, de acordo com o tratamento previsto, perfazendo um total de 1 hora e 24 horas de observação.

Os seguintes parâmetros foram observados: presença de sinais colinérgicos periféricos (SCP - miose, piloereção, cromodacriorréia, salivação, diarréia e diurese), tremores, movimentos estereotipados (aumento da atividade de roer, coçar, mastigar e wet-dog shakes (ato de sacudir - semelhante a um cachorro molhado)), convulsões motoras (incluindo movimentos clônicos das extremidades superiores que ocorrem em aproximadamente 30 minutos após administração da pilocarpina), que progrediram para o estado epiléptico 
Freitas, R. M. et al./Revista Eletrônica de Farmácia Vol 5(3), 60 - 70, 2008.

(convulsões motoras límbicas foram definidas como contínuas quando ocorrem por um período maior que 30 minutos) e o número de animais que morreram em cada grupo. Depois desse período de observação, os animais foram sacrificados por decapitação e os cérebros removidos para a dissecação do hipocampo, corpo estriado e córtex frontal para a realização dos estudos neuroquímicos.

\section{Determinação da atividade enzimática da Superóxido Dismutase (SOD)}

A atividade da SOD foi determinada através da técnica descrita anteriormente por FLOHÉ \& OTTING, 1984. Foi preparado o meio da reação contendo tampão fosfato de potássio $50 \mathrm{mM}, \mathrm{pH} 7,8$, xantina $500 \mu \mathrm{M}$; cianeto de potássio $200 \mu \mathrm{M}$; EDTA $1 \mathrm{mM}$. Os homogenatos (10\%) foram preparadas com tampão fosfato de potássio $50 \mathrm{mM}, \mathrm{pH} 7,8$. Em seguida centrifugados a $15000 \mathrm{rpm}$ durante $15 \mathrm{~min}$ a $4^{\circ} \mathrm{C}$. Os sobrenadantes foram removidos para determinação da atividade da SOD.

A xantina oxidase $(\mathrm{XO})$ usada na reação foi preparada a partir da solução padrão de $\mathrm{XO}$ em tampão fosfato de potássio $50 \mathrm{mM}, \mathrm{pH} 7,8$. Em seguida foi realizado o ensaio contendo $975 \mu \mathrm{L}$ do meio reacional, $20 \mu \mathrm{L}$ do da amostra e $5 \mu \mathrm{L}$ da XO, a mistura foi agitada e em seguida realizada a leitura a $550 \mathrm{~nm}$ durante 6 minutos. A leitura do branco cinético foi feita a $550 \mathrm{~nm}$. A quantidade da atividade da SOD das amostras foi calculada usando a média das absorções lineares obtidas durante 6 min pela curva.

Os resultados foram expressos em U/mg de proteína. A concentração da proteína foi obtida pelo método de Lowry et al., 1951.

\section{Determinação da atividade enzimática da Catalase (CAT)}

A atividade da catalase foi determinada de acordo com o método descrito anteriormente por CHANCE \& MAEHLY, (1955). Foi preparado o meio da reação com $\mathrm{H}_{2} \mathrm{O}_{2}$ em Tampão Tris $\mathrm{HCl} 1 \mathrm{M}$, EDTA $5 \mathrm{mM} \mathrm{pH} 8,0$ e $\mathrm{H}_{2} \mathrm{O}$ ultrapurificada. Em seguida foi colocado $980 \mu \mathrm{L}$ do meio de reação e $20 \mu \mathrm{L}$ do homogenato a $10 \%$ preparado em tampão fosfato de sódio $50 \mathrm{mM}, \mathrm{pH} 7,4$. A leitura foi feita durante 6 min a $230 \mathrm{~nm}$. Como branco cinético foi feito

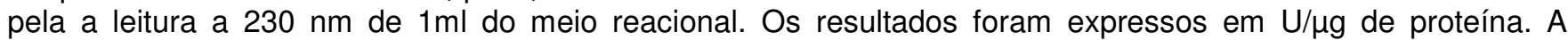
concentração de proteína foi determinada pelo método de Lowry et al., 1951.

\section{Análise Estatística}

Os resultados que obedeciam a uma distribuição paramétrica foram analisados por Análise de Variância (ANOVA) com teste de Student Newman Keuls (post hoc) pelo programa GraphPad Prism versão 3.00 para Windows, GraphPad Software, San Diego California USA. Copyright (c) 1994-1999 por GraphPad software. O mesmo programa (GraphPad Prism(C) foi utilizado para confecção dos gráficos apresentados neste trabalho. As diferenças foram consideradas estatisticamente significativas a partir de $p<0,05$.

\section{RESULTADOS}

Os estudos comportamentais foram realizados como descrito anteriormente. Todos os animais adultos tratados com dose elevada de pilocarpina $(400 \mathrm{mg} / \mathrm{kg}, \mathrm{sc} ; \mathrm{n}=40)$, durante 1 hora de observação, apresentaram sinais colinérgicos periféricos (SCP), tais como, miose, piloereção, cromodacriorréia, salivação, diarréia, diurese, e também movimentos estereotipados (ME), envolvendo o aumento da atividade de roer, coçar, mastigar e wetdog shakes (ato de sacudir - semelhante a um cachorro molhado). Os tremores ocorreram em $50 \%$ (20) e as convulsões em 58\% (23) que apareceram nos 20 minutos iniciais, sendo instalado o estado de mal epiléptico logo em seguida, em 55\% (22) dos animais, não havendo nenhuma morte nesse grupo. Por sua vez, durante o período de 24 horas de observação, todos os animais (40) apresentaram também SCP, ME e tremores. As convulsões ocorreram em 75\% (30) dos animais, que progrediram para o estado de mal epiléptico em $75 \%$ (30) dos mesmos. Neste grupo foi observado $63 \%$ (25) de morte.

Foi observado um aumento de $24 \%$ (controle $=2,35 \pm 0,14 ; \mathrm{P} 4001 \mathrm{~h}=2,91 \pm 0,06$ ), $[\mathrm{p}<0,0047] \mathrm{da}$ atividade da enzima no hipocampo dos animais que apresentaram convulsões, estado epiléptico e foram sacrificados $1 \mathrm{~h}$ após o tratamento. No grupo sacrificado após 24 horas não foi verificada alteração no valor da atividade da SOD em relação ao grupo controle (controle $=2,35 \pm 0,14 ; \mathrm{P} 40024 \mathrm{~h}=2,45 \pm 0,10$ ), $[\mathrm{p}=\mathrm{N}$.S.]. Com relação à atividade enzimática no corpo estriado dos ratos adultos foi observado um aumento significativo no período de $1 \mathrm{~h}$ (controle $=3,25 \pm 0,06 ; \mathrm{P} 4001 \mathrm{~h}=4,23 \pm 0,14$ ), [p<0.0001] e após $24 \mathrm{~h}$ de observação não houve alteração (controle $=3,25 \pm 0,06 ; \mathrm{P} 40024 \mathrm{~h}=3,23 \pm 0,19$ ), [p=N.S.] (Figura 1).

Por sua vez, a atividade da SOD também foi estudada no córtex frontal de ratos adultos e verificou-se um aumento significativo de 72 e $24 \%$ nos grupos observados após $1 \mathrm{~h}$ (controle $=2,12 \pm 0,09 ; \mathrm{P} 4001 \mathrm{~h}=3,65 \pm$ 
Freitas, R. M. et al./Revista Eletrônica de Farmácia Vol 5(3), 60 - 70, 2008.

0,04), $[p<0.0001]$ e $24 \mathrm{~h}$ (controle $=2,12 \pm 0,09 ; \mathrm{P} 40024 \mathrm{~h}=2,62 \pm 0,16),[p<0.0100]$ do tratamento com pilocarpina em alta dose, respectivamente.

E quando comparados os grupos tratados observou-se uma redução na atividade da SOD de $28 \%$ no grupo 24h em relação ao de $1 \mathrm{~h}$ (P400 1h =3,65 $\pm 0,04 ; \mathrm{P} 40024 \mathrm{~h}=2,62 \pm 0,16)$, [p<0.0001] (Figura 1).

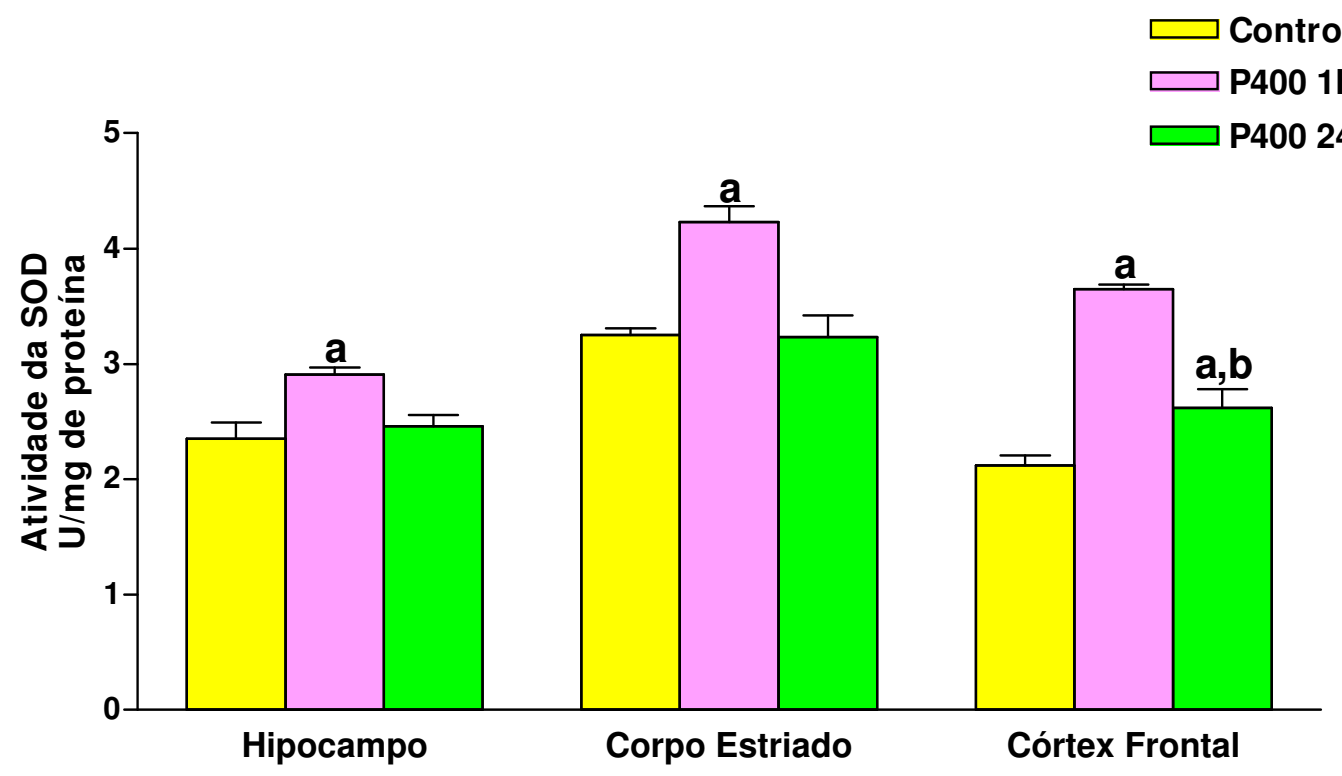

Figura 1 - A atividade enzimática da superóxido dismutase (SOD) em diferentes áreas cerebrais (hipocampo, corpo estriado e córtex frontal) de ratos adultos durante a fase aguda do processo convulsivo induzido por pilocarpina.

Ratos Wistar (250-280g, 2 meses de idade) foram tratados com uma única dose de pilocarpina (400 mg/kg, s.c., $n=8)$ e os controles com salina $0,9 \%(n=10)$. Os animais estudados apresentaram convulsão, estado epiléptico e foram sacrificados 1 e $24 \mathrm{~h}$ após o tratamento. Os valores representam à média \pm EPM. Para análise estatística foram usados ANOVA e teste $t$ Student Newman Keuls como post hoc.

a quando os grupos tratados com pilocarpina (P400 1 e 24h) foram comparados ao grupo controle $(p<0,05)$;

'quando o grupo tratado com pilocarpina (P400 24h) foi comparado ao grupo P400 $1 \mathrm{~h}(p<0,05)$.

Um aumento de 32 e $80 \%$ foi observado na atividade da enzima CAT no hipocampo dos animais que apresentaram convulsões, estado epiléptico e foram sacrificados $1 \mathrm{~h}$ (controle $=14,50 \pm 0,65 ; \mathrm{P} 4001 \mathrm{~h}=19,78 \pm$ 0,75), $[\mathrm{p}<0,0001]$ e $24 \mathrm{~h}$ (controle $=14,50 \pm 0,65 ; \mathrm{P} 40024 \mathrm{~h}=27,25 \pm 1,03$ ), [p<0,0001], após o tratamento, respectivamente. No grupo sacrificado após 24 horas foi verificado também um aumento de $38 \%$ em relação ao grupo 1h (P400 1h = 19,78 $\pm 0,75 ; \mathrm{P} 40024 \mathrm{~h}=27,25 \pm 1,03)$, $[\mathrm{p}<0,0001]$ (Figura 2).

A atividade enzimática da CAT também foi verificada no corpo estriado dos ratos adultos após o tratamento, e foi visto um aumento de 29 e $36 \%$ após os períodos de $1 \mathrm{~h}$ (controle $=13,66 \pm 0,77 ; \mathrm{P} 4001 \mathrm{~h}=$ $17,97 \pm 0,09),[p<0.0001]$ e $24 \mathrm{~h}$ de observação em relação ao controle (controle $=13,66 \pm 0,77 ; \mathrm{P} 40024 \mathrm{~h}=$ $19,05 \pm 0,06),[p<0.0001]$.

Quando comparados os grupos tratados evidenciou-se um aumento de $6 \%$ nos animais sacrificados após $24 \mathrm{~h}$ em relação ao de $1 \mathrm{~h}$ (P400 $1 \mathrm{~h}=17,97 \pm 0,09 ; \mathrm{P} 40024 \mathrm{~h}=19,05 \pm 0,06),[p<0.0001]$. Por sua vez, a atividade da CAT no córtex frontal demonstrou um aumento de 28 e $49 \%$ após os períodos de $1 \mathrm{~h}$ (controle $=$ $22,15 \pm 1,63 ; \mathrm{P} 4001 \mathrm{~h}=28,32 \pm 2,00$ ), [ $\mathrm{p}<0.0291]$ e $24 \mathrm{~h}$ de observação em relação ao controle (controle $=22,15$ $\pm 1,63 ; \mathrm{P} 40024 \mathrm{~h}=32,93 \pm 0,18),[\mathrm{p}<0.0001]$. E quando comparados os grupos tratados, evidenciou-se um aumento de $16 \%$ nos animais sacrificados após $24 \mathrm{~h}$ em relação ao de $1 \mathrm{~h}(\mathrm{P} 4001 \mathrm{~h}=28,32 \pm 2,00 ; \mathrm{P} 40024 \mathrm{~h}=$ $32,93 \pm 0,18),[p<0.0268]$ (Figura 2). 
Freitas, R. M. et al./Revista Eletrônica de Farmácia Vol 5(3), 60 - 70, 2008.

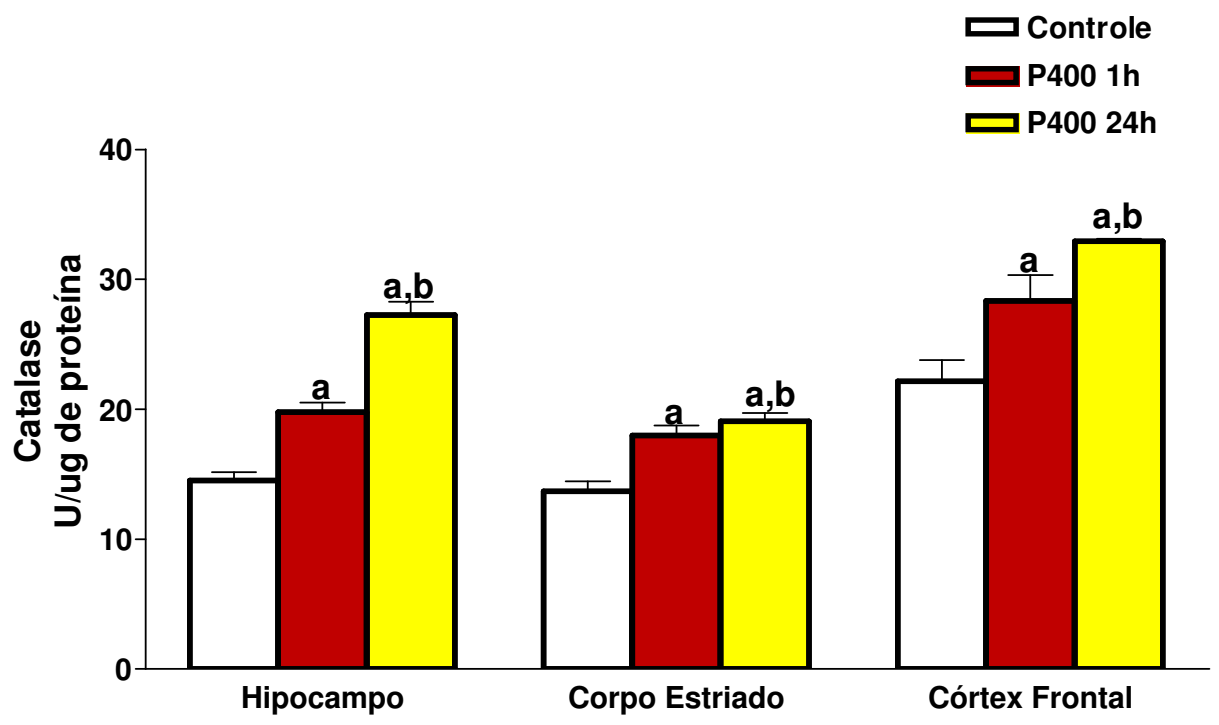

Figura 2 - A atividade enzimática da catalase em diferentes áreas cerebrais (hipocampo, corpo estriado e córtex frontal) de ratos adultos durante a fase aguda do processo convulsivo induzido por pilocarpina.

Ratos Wistar (250-280g, 2 meses de idade) foram tratados com uma única dose de pilocarpina (400 mg/kg, s.c., $n=8)$ e os controles com salina $0,9 \%(n=9)$. Os animais estudados apresentaram convulsão, estado epiléptico e foram sacrificados 1 e $24 \mathrm{~h}$ após o tratamento. Os valores representam à média \pm EPM. Para análise estatística foram usados ANOVA e teste $t$ Student Newman Keuls como post hoc.

${ }^{a}$ quando os grupos tratados com pilocarpina ( $P 4001$ e $\left.24 \mathrm{~h}\right)$ foram comparados ao grupo controle $(p<0,05)$;

bquando o grupo tratado com pilocarpina (P400 24h) foi comparado ao grupo P400 1h ( $p<0,05)$.

Modelos de convulsão induzidos em animais reproduzem alterações comportamentais e eletroencefalográficas que são semelhantes à epilepsia do lobo temporal de humanos (CAVALHEIRO et al., 1991). O modelo de convulsão induzido por pilocarpina em alta dose $(400 \mathrm{mg} / \mathrm{kg})$ foi utilizado como instrumento para investigar as alterações comportamentais, e a atividade das enzimas SOD e CAT durante a fase aguda do processo convulsivo. A convulsão induzida por pilocarpina pode ser caracterizada por episódios de alteração no comportamento dos animais (TURSKI et al., 1983). A atividade epiléptica pode ser detectada pelas alterações eletroencefalográficas e comportamentais nos animais convulsivos (CAVALHEIRO et al., 1991).

Imediatamente após a administração de pilocarpina, os ratos podem apresentar persistentes mudanças comportamentais, incluindo piloereção, cromodacriorréia, acinesia, ataxia, tremores, automatismos mastigatórios como mioclonia dos músculos faciais, e movimentos de cachorro molhado, que persistem de 10 a 15 minutos (TURSKI et al., 1983). Estas alterações comportamentais prosseguem para convulsões motoras límbicas incluindo movimentos clônicos das extremidades superiores que ocorrem em aproximadamente 30 minutos após administração da pilocarpina, e ainda pode desenvolver estado epiléptico de longa duração no animal (12-18h). As convulsões motoras límbicas foram definidas como contínuas quando ocorrem por um período maior que 30 minutos, sendo este parâmetro utilizado para indicar a presença do estado epiléptico (EP) em nossos animais tratados com pilocarpina (SZYNDLER et al., 2005).

Cavalheiro e colaboradores (1994) observaram também que essas convulsões límbicas podem persistir por aproximadamente 60 minutos, desenvolvendo em $75 \%$ dos ratos convulsivos o EP com retorno progressivo ao normal depois de 3-5 horas e permanecendo em praticamente todos os animais tratados com pilocarpina. $O$ EP pode persistir por aproximadamente 12 a 18 horas e 30\% dos ratos em EP morrem durante este período.

Nossos resultados indicam que os animais tratados com uma única dose convulsiva de pilocarpina apresentaram as características semelhantes às descritas por MARINHO e colaboradores, (1997), confirmando a existência das alterações comportamentais após a administração do estímulo convulsivo.

Todos os animais observados por 1 e $24 \mathrm{~h}$ apresentaram SCP e ME. Após 35 minutos da administração de pilocarpina, surgiram às convulsões motoras límbicas nos animais observados por 1 e $24 \mathrm{~h}$, e em seguida as convulsões recorrentes foram instaladas em $50 \%$ e $75 \%$ dos animais, respectivamente.

Em relação ao número de mortes dos animais, foi visto que no grupo de $1 \mathrm{~h}$ nenhum animal morreu, enquanto, que no grupo observado durante $24 \mathrm{~h}, 63 \%$ dos animais morreram. O número de animais usado em cada grupo experimental pode ser justificado pelo fato de não estar estabelecido no modelo de convulsão induzido por pilocarpina em alta dose o índice de mortalidade durante a fase aguda das convulsões límbicas, o que permitiu após os períodos de observação à determinação desses índices em função destes como descrito 
Freitas, R. M. et al./Revista Eletrônica de Farmácia Vol 5(3), 60 - 70, 2008.

anteriormente. Estas observações são semelhantes às características comportamentais expressas por Clifford e colaboradores (1987) que determinaram um percentual de $81 \%$ de morte dos animais com EP.

Observa-se, portanto, que no processo convulsivo há um aumento significativo no número de morte dos animais em função do período de tempo observado da fase aguda, mostrando, assim, uma possível relação entre o tempo e o estabelecimento das mortes dos animais adultos durante o desenvolvimento do processo convulsivo, o que pode sugerir uma maior interação entre os diferentes sistemas de neurotransmissão cerebral em decorrência da propagação e manutenção da atividade epiléptica, levando a uma possível potencialização dos efeitos neurotóxicos sobre o SNC.

Algumas pesquisas procuram conhecer e esclarecer melhor à fisiopatologia do processo convulsivo, procurando detectar as mudanças em nível neuroquímico (SIMONIC et al., 2000) em diferentes estruturas cerebrais, bem como demonstrar o possível envolvimento genético no controle e estabelecimento da atividade epiléptica em humanos e em animais de experimentação (VANHATALO \& RIIKONEN, 2001), a fim de contribuir para o pronto esclarecimento dos mecanismos de diferentes modelos de epilepsia.

Na tentativa de esclarecer a fisiopatologia da epilepsia, faz-se necessário investigar duas enzimas (SOD e CAT), que podem encontrar se alteadas durante o estresse oxidativo (EO) cerebral no animal convulsivo. $O$ cérebro é mais vulnerável ao EO que outros tecidos, por que contém uma grande quantidade de lipídios e metais oxidáveis, e tem comparado a outros tecidos, menos mecanismos de defesa antioxidantes (HYND, 2004).

Durante o EO são produzidos radicais livres $(\mathrm{RL})$ tais como superóxido $\left(\mathrm{O}_{2}^{-}\right)$e radical hidroxila $\left(\mathrm{OH}^{-}\right)$. Estes radicais têm tem sido implicados em uma variedade de processos degenerativos, doenças e síndromes. Algumas destas doenças incluem infarto do miocárdio, isquemia cerebral, condições inflamatórias agudas e crônicas e desordens do SNC como doença de Parkinson, epilepsia e demência (Alzheimer's). Em todos esses eventos patológicos ocorrem inúmeras alterações bioquímicas como, por exemplo, o da produção do íon superóxido e da expressão das isoformas e da atividade da SOD (HYND, 2004).

Os RL são compostos químicos caracterizados por um orbital contendo elétrons não pareados. Estes conferem aos RL uma capacidade peculiar de interagir com um elétron de outras moléculas com um orbital completo, produzindo dano à estrutura que o RL se ligou (CASTAGNE et al. 1999). Um RL de grande importância em processos patológicos é o superóxido $\left(\mathrm{O}_{2}^{-}\right)$, que pode ser gerado no cérebro por vários mecanismos, a saber: ineficiência do transporte de elétrons durante o ciclo de Krebs nas mitocôndrias, metabolização das monoaminas e aminoácidos, reação da xantina oxidase, pelo metabolismo do ácido araquidônico e durante o desenvolvimento do processo convulsivo (NAFFAH-MAZZACORATTI et al., 2001). Esse radical produzido pode ser metabolizado pelas enzimas superóxidos dismutases (SODs) que estão presentes no citoplasma (zinco-isoforma) e na mitocôndria (magnésio-isoforma) (NAFFAH-MAZZACORATTI et al., 2001).

Investigou-se, assim, a atividade da SOD nas diferentes áreas cerebrais (córtex frontal, corpo estriado e hipocampo) durante 1 e $24 \mathrm{~h}$ da fase aguda da convulsão induzida por pilocarpina. Verificou-se, um aumento da atividade da SOD em todas as áreas cerebrais durante a primeira hora da convulsão. Enquanto que, no grupo observado por $24 \mathrm{~h}$ a atividade enzimática aumentou apenas no córtex frontal em relação às demais áreas. As mudanças na atividade enzimática, nos experimentos "in vitro", revelaram que, no processo convulsivo, pode haver a interferência na atividade da enzima, apenas de forma aguda e a curto prazo em todo o cérebro, uma vez que, foi observado a ausência de mudanças após $24 \mathrm{~h}$ da administração do estímulo convulsivo em duas das três áreas investigadas.

Alguns estudos sugerem que o EO pode ter uma importante participação na etiologia da convulsão, induzindo morte neuronal nos animais adultos (CHAN \& MAIER, 2002) e que facilita a progressão do quadro convulsivo, além de permitir o aparecimento de crises convulsivas recorrentes. O EO pode ser produzido através da quebra do balanço entre a formação de EROs e da atividade das enzimas antioxidantes (SOD, catalase e glutationa peroxidase (GPx). O mediador central do EO pode ser o radical superóxido, que influencia ambos os processos fisiológicos e patológicos no cérebro de humanos e ratos (HONG et al., 2000). $\mathrm{O}_{2}^{-}$danifica diretamente uma minoria de componentes celulares, como exemplo proteínas Fe-S, mas é um importante precursor de compostos oxidantes, tais como: radicais hidroxilas $\left(\mathrm{OH}^{\circ}\right)$ e peroxinitrito (ONOO'), nitrito e nitrato. $A$ SOD catalisa a conversão de $\mathrm{O}_{2}^{-}$em $\mathrm{H}_{2} \mathrm{O}_{2}$ e $\mathrm{O}_{2}$, mantendo, assim, baixos, os níveis de $\mathrm{O}_{2}^{-}$que não induzem o aparecimento de patologias em humanos (LIU et al., 2002).

Da mesma forma em que não foi observada alteração na atividade da SOD na maioria das áreas cerebrais em nosso estudo, após $24 \mathrm{~h}$ da fase aguda, Karakoc e colaboradores (2002) também não evidenciaram mudanças na atividade desta enzima em pacientes epilépticos jovens. Por outro lado, foi visto um aumento nos níveis de lipídios peróxidos nesses pacientes de forma semelhante ao aumento observado nas três regiões, e durante as duas horas investigadas da fase aguda das convulsões límbicas (dados não mostrados). Assim, os sistemas antioxidantes e os níveis de lipoperoxidação podem não estar correlacionados com a atividade epiléptica pelo menos durante $24 \mathrm{~h}$ da fase aguda. Embora, tenha sido verificado um aumento na atividade da SOD durante a primeira hora da convulsão foi verificado também um aumento no conteúdo de lipídio peróxidos, sugerindo que ativação desta enzima pode não ter sido suficiente para exercer um papel fundamental de proteção contra danos na membrana neuronal nas áreas analisadas. Outros mecanismos antioxidantes, tais como: catalase e GPx, podem ser necessários para realizar um papel neuroprotetor auxiliando a remoção de RL durante o estabelecimento e propagação das convulsões. A atividade da SOD encontra-se normal em eventos 
Freitas, R. M. et al./Revista Eletrônica de Farmácia Vol 5(3), 60 - 70, 2008.

fisiológicos e parece participar dos mecanismos celulares e moleculares do dano cerebral e de outras anormalidades neurológicas, inclusive da epilepsia.

A SOD é uma das mais importantes defesas antioxidantes nas diferentes estruturas cerebrais e em outros tecidos, realizando uma desintoxicação e protegendo a membrana contra danos em diferentes modelos de convulsão (SIMONIC et al., 2003). Por sua vez, entre as enzimas antioxidantes acredita-se que no hipocampo a principal delas pode ser a SOD, já que esta área cerebral contém uma grande quantidade de zinco e cobre e que superexpressão da CuZn-SOD em camundongos transgênicos pode atenuar 0 dano oxidativo e a neurotoxicidade hipocampal (MURAKAMI et al., 1997) reduzindo os malefícios da atividade epiléptica.

A catalase é uma hemeproteína citoplasmática que catalisa a redução do peróxido de hidrogênio $\left(\mathrm{H}_{2} \mathrm{O}_{2}\right)$, juntamente com a glutationa peroxidase em água e oxigênio (SIMONIC et al., 2000). É importante na manutenção do metabolismo normal das EROs, sendo seu papel bastante relevante para o funcionamento celular em diferentes partes do organismo (NAFFAH-MAZZACORATTI et al., 2001).

Inúmeras EROs são normalmente produzidas em diferentes áreas do cérebro, tais como superóxido e radical hidroxila, e além disso, ocorre a produção de peróxido de hidrogênio $\left(\mathrm{H}_{2} \mathrm{O}_{2}\right)$ durante o catabolismo das mesmas. $\mathrm{O} \mathrm{H}_{2} \mathrm{O}_{2}$, por si só não é um $\mathrm{RL}$, mas em alta concentração pode reagir com o íon superóxido (Reação de Haber-Weiss) ou com o ferro (Reação de Fenton) produzindo um novo radical livre, o radical hidroxila (OH') que é altamente reativo e pode induzir diferentes danos teciduais ao reagir com macromoléculas protéicas.

Os resultados do presente estudo mostraram um aumento da atividade da catalase no hipocampo, corpo estriado e córtex frontal de ratos que apresentaram convulsão, estado epiléptico e que foram sacrificados após a primeira hora de observação (FREITAS et al., 2004a). No grupo sacrificado, após 24 h da fase aguda, a atividade desta enzima também estava aumentada nas áreas investigadas, sugerindo, uma maior participação desta em relação à outra enzima investigada (SOD) durante a atividade epiléptica em função do período da fase aguda das convulsões límbicas induzidas por pilocarpina.

As convulsões induzidas por pilocarpina produzem uma série de mudanças em parâmetros relacionados com a produção e eliminação de EROs (SIMONIC et al., 2000). Um aumento na formação dos RL pode ser acompanhado por um mecanismo compensatório de aumento na atividade das enzimas removedoras de $R L$ e esta ação foi observada após convulsão e EP no hipocampo, córtex frontal e corpo estriado de ratos adultos, e este aumento pode estar relacionado como um mecanismo compensatório em função do tempo, através da modulação da atividade das enzimas envolvidas no metabolismo dos RLs (FREITAS et al., 2004b).

Em adição, verificam-se durante o funcionamento fisiológico cerebral mudanças na atividade neuronal que são acompanhadas por alterações no metabolismo cerebral induzindo modificações no fluxo sanguíneo cerebral (DYMOND et al., 1976; WALZ et al., 2000) prejudicando o seu funcionamento fisiológico cerebral. Em contraste, durante distúrbios patológicos, o fluxo sanguíneo pode não ocorrer desta maneira. Evidências clínicas e experimentais demonstraram possíveis alterações no nível basal do oxigênio durante e após as convulsões, verificadas pela redução da concentração do oxigênio; simultaneamente, um elevado fluxo sanguíneo foi observado, provavelmente devido a uma vasodilatação secundária (DYMOND et al., 1976).

Considerando que um aumento da demanda metabólica pode ser observado durante as convulsões límbicas, pode-se sugerir que atividade da catalase poderia ser uma das enzimas que teria sua atividade inalterada, aumenta ou diminuída durante a atividade epiléptica. Em nossos ensaios bioquímicos foi verificado um aumento em todas as áreas analisadas na atividade da catalase, porém, em uma outra área estudada, o cerebelo, não foi verificada nenhuma mudança significativa (dados não mostrados), sugerindo, ainda, que nas outras áreas que não foram estudadas nas mesmas condições, o metabolismo das EROs pode permanecer pelo menos em parte inalterado após a convulsões e EP induzido por pilocarpina (FREITAS et al., 2004b).

A evidência da participação da EROs durante as convulsões tem sido sugerida através da administração exógena de antioxidantes enzimáticos e não enzimáticos para possível proteção contra o dano neuronal induzido pelas convulsões e EP (LAPIN et al., 1998). O fato de que a atividade da catalase está aumentada pode não resultar em efeitos neurotóxicos durante o EP induzido pela pilocarpina, indica que a produção alterada de EROs pode danificar neurônios e precisa ser controlada (NAFFAH-MAZZACORATTI et al., 2001). Precisa ser esclarecido o seu envolvimento das EROs em fenômenos neurodegenerativos, tais como a convulsão. A relação entre a atividade da catalase e a primeira fase das convulsões tem sido mais proposta do que demonstrada, por que existem certas dificuldades em estabelecê-la (NAFFAH-MAZZACORATTI et al., 2001). A maior dificuldade está em demonstrar se o aumento funcional na modulação da atividade desta enzima é a causa ou conseqüência das convulsões.

Correa e colaboradores (2001) determinaram aumentos na atividade da catalase cerebral e na atividade locomotora nos camundongos tratados com álcool. Em nossos estudos comportamentais foi possível verificar em testes de atividade locomotora (dados não mostrados) que durante a primeira hora da convulsão ocorre diminuição desta, entretanto, já atividade da catalase aumenta, sugerindo que pode não haver interação entre a estimulação motora e atividade enzimática da catalase no processo convulsivo ao contrário do que ocorre com uso de substâncias depressoras. No entanto, futuros estudos devem ser realizados para melhor esclarecer esta hipótese. 
Freitas, R. M. et al./Revista Eletrônica de Farmácia Vol 5(3), 60 - 70, 2008.

\section{CONSIDERAÇÕES FINAIS}

Nossos resultados indicam que as convulsões induzidas por pilocarpina apresentam alterações comportamentais características da atividade epiléptica e pode haver também um incremento considerável nas atividades das defesas antioxidantes, entre elas SOD, pelo menos na primeira hora da fase aguda, e na catalase nos dois períodos estudados durante a fase aguda no cérebro de ratos adultos, na tentativa de proteger o animal contra o dano neuronal observado nas estruturas cerebrais estudadas. A anatômica distribuição das alterações nas atividades da SOD e catalase observada sugere uma extensa participação do hipocampo, do corpo estriado e do córtex frontal de ratos adultos na instalação, propagação e/ou manutenção do processo convulsivo induzido por pilocarpina.

Novos estudos serão realizados para se avaliar de forma mais detalhada a atividade das enzimas (SOD e CAT) em outras áreas cerebrais e em novos períodos de observação (fase silenciosa e crônica), além da aguda, já estudada no presente trabalho, com o propósito de contribuir para o esclarecimento da fisiopatologia das convulsões límbicas induzidas pela pilocarpina que são semelhantes à epilepsia do lobo temporal de humanos.

\section{REFERÊNCIAS BIBLIOGRÁFICAS}

BEN-ARI, Y.; TREMBLAY, E.; RICHE, D.; GHILINI, G.; NAQUET, R. Electrographic, clinical and pathological alterations following systemic administration of kainic acid, bicuculine, deoxyglucose or pentylenetetrazole: metabolite mapping using the deoxyglucose method with special reference to the pathology of epilepsy. Neuroscience. v. 6, p. 1361-1391, 1981.

BORELLI, E.; BOZZI, Y. Dopamine $\mathrm{D}_{2}$ receptor signaling controls neuronal cell death induced by muscarinic and glutamatergic drugs. Molecular and Cellular Neuroscience. v. 19, p. 263-271, 2002.

BOVERIS, A.; CADENAS, E.; REITER, R.; CHANCE, B.; JAMIESON, D. The relation of free radical production to hyperoxia. Annuals Revision Physiology. v. 48, p. 703-719, 1986.

CASTAGNE, V.; GASTSCHI, M.; LEFEVRE, K.; POSADA, A.; CLARKE, P.G.H. Relationship between neuronal death and cellular redox status, focus on the developing nervous system. Progress Neurophysiology. v. 59, 397423, 1999.

CAVALHEIRO, E.A.; LEITE, J.P.; BORTOLOTTO, Z.A.; TURSKI, W.A.; IKONOMIDOU, C.; TURSKI, L. Long-term effects of pilocarpine in rats: structural damage of the brain triggers kindling and spontaneous recurrent seizures. Epilepsia. v. 32, p. 778-782, 1991.

CAVALHEIRO, E.A.; FERNANDES, M.J.; TURSKI, L.; NAFFAH-MAZZACORATTI, M.G. Spontaneous recurrent seizures in rats: amino acid and monoamine determination in the hippocampus. Epilepsia. v. 35, p. 1-11, 1994.

CAVALHEIRO, E.A.; BELLÍSSIMO, M.I.; NAFFAH-MAZZACORATTI, M.G. Profile of prostaglandin levels in the rat hippocampus in pilocarpine model of epilepsy. Neurochemical. v. 27, n. 6, p. 461-466, 1995.

CHANCE, B.; MAEHLY, A.C. Assay catalases and peroxidases. Methods Enzymolology. v. 2, p. 764-768, 1955.

CHAN, P.H.; MAIER, C.M. Role of superoxide dismutases in oxidative damage and neurodegenerative disorders. Neuroscience. v. 8, n. 4, p. 323-334, 2002

CLIFFORD, D.B.; OLNEY, J.W.; MANIOTIS, A.; COLLINS, R.C.; ZORUMSKI, C.F. The functional anatomy and pathology of lithium-pilocarpine and high-dose pilocarpine seizures. Neuroscience. v. 23, p. 953-968, 1987.

CORREA, M.; SANCHIS-SEGURA, C.; ARAGON, C.M.G. Brain catalase activity in highly correlated with ethanolinduced locomotor activity in mice. Physiology \& Behavior. v. 73, p. 641-647, 2001.

COSTA-LOTUFO, L.V.; FONTELES, M.M.F.; LIMA, I.S.P.; OLIVEIRA, A.A.; NASCIMENTO, V.S.; BRUIN, V.M.S.; VIANA, G.S.B. Attenuating effects of melatonin on pilocarpine-induced seizures in rats. Comparative Biochemistry and Physiology Part C. v. 131, p. 521-529, 2002. 
Freitas, R. M. et al./Revista Eletrônica de Farmácia Vol 5(3), 60 - 70, 2008.

De LORENZO, R. J.; CHURN, S. B.; KOCHAN, L. D. Chronic inhibition of $\mathrm{Ca}^{2+} /$ Calmodulin kinase II activity in the pilocarpine model of epilepsy. Brain Research. v. 875, p. 66-77, 2000.

De LORENZO, R.J.; RAZA, M.; PAL, S.; RAFIQ, A. Long-term alteration of calcium homeostastic mechanisms in the pilocarpine model of temporal lobe epilepsy. Brain Research. v. 903, p. 1-12, 2001.

DYMOND, A. M.; CRANDALL, P.H. Oxygen availability and blood flow in the temporal lobes during spontaneous epileptic seizures in men. Brain Research. v. 102, p. 191-196, 1976.

DODRILL, C.B. Neuropsychological effects of seizures. Epilepsy \& Behavior. v. 5, p. S21-S24, 2004.

FRANTSEVA, M.V.; PEREZ VELAZQUEZ, J.L.; HWANG, P.A.; CARLEN, P.L. Free radical production correlates with cell death in an in vitro model of experimental epilepsy. Epilepsy Research. v. 9, p. 6-71, 2000.

FREITAS, R.M.; SOUSA, F.C.F.; VASCONCELOS, S.M.M.; VIANA, G.S.B.; FONTELES, M.M.F. Pilocarpineinduced seizures in adult rats: lipid peroxidation level, nitrite formation, GABAergic and glutamatergic receptor alterations in the hippocampus, striatum and frontal cortex. Pharmacology Biochemistry Behavior. v. 78, n. 2, p. 327-332, 2004a.

FREITAS, R.M.; NASCIMENTO, V.S.; SOUSA, F.C.F.; VASCONCELOS, S.M.M.; VIANA, G.S.B.; FONTELES, M.M.F. Catalase activity in cerebellum, hippocampus, frontal cortex and striatum after status epilepticus induced by pilocarpine in Wistar rats. Neuroscience Letters. v. 365, n. 2, p. 102-105, 2004 b.

FLOHE L.; OTTING F. Superoxide dismutase assays. Methods Enzymology. v. 105, p. 93-104, 1984.

HALLIWELL, B.; GUTTERIDGE, J.M.C. Free radicals, antioxidants and human disease: where are we now? Journal Laboratory Clinical Medical. v. 119, p. 598-620, 1992.

HONG, J.; KATO, K.; KIM, S.J.; SUH, J.H.; KIM, W.K.; HO KO, K.; JHOO, W.K.; BING, G.; KIM, H.C. Changes of hippocampal $\mathrm{Cu} / \mathrm{Zn}$-superoxide dismutase after kainite treatment in the rat. Brain Research. v. 853, p. 215-226, 2000.

HYND, M.R.; SCOTT, H.L.; DODD, P.R. Glutamate-mediated excitotoxicity and neurodegeneration in Alzheimer's disease. Neurochemistry International. v. 45, p. 583-595, 2004.

KARAKOC, Y.; TOPLAN, S.; TURKDOGAN, D. Lipid peroxidation and antioxidative enzyme activities in childhood epilepsy. Journal Children Neurology. v. 17, n.9, p. 673-676, 2002.

KEHER, J.P. Free radicals as mediators of tissue injury and disease. Critical Revist Toxicology. v. 114, n. 1, p. 2148, 1993.

LAPIN, I.P.; MIRZAEV, S. M.; RYZOV, I.V.; OXENKRUG, G.F. Anticonvulsant activity of melatonin against seizures induced by quinolinate, kainate, glutamate, NMDA, and pentylenetetrazol in mice. Journal Pineal Research. v. 24, p. 215-218, 1998.

LIU, K.J.; LIU, S.; MORROW, D.; PETERSON, S.L. Hydroethidine detection of superoxide production during the lithium-pilocarpine model of status epilepticus. Epilepsy Research. v. 49, p.226-238, 2002.

LOWRY, O.H., ROSEBROUGH, N.J., FARR, A.L., RANDAL, R.J. Protein measurement with follin phenol reagent. Journal Biological Chemistry. v. 193, p. 265-275, 1951.

MARINHO, M.M.F.; SOUSA, F.C.F.; BRUIN, V.M.S.; AGUIAR, L.M.V.; PINHO, R.S.N.; VIANA, G.S.B. Inhibitory action of a calcium channel blocker (nimodipine) on seizures and brain damage induced by pilocarpine and lithiumpilocarpine in rats. Neuroscience Letters. v. 235, p. 13-16, 1997.

MARINHO, M.M.F.; SOUSA, F.C.F.; BRUIN, V.M.S.; VALE, M.R.; VIANA, G.S.B. Effects of lithium, alone or associated with pilocarpine, on muscarinic and dopaminergic receptors and on phosphoinositide metabolism in rat hippocampus and striatum. Neurochemisty International. v. 33, p. 299-306, 1998. 
Freitas, R. M. et al./Revista Eletrônica de Farmácia Vol 5(3), 60 - 70, 2008.

MICHOTTE, Y.; KHAN, G.M.; SMOLDERS, I.; EBINGER, G. Anticonvulsant effect and neurottransmitter modulation of focal and systemic 2-chloroadenosine against the development of pilocarpine-induced seizures. Neuropharmacology. v. 39, p. 2418-2432, 2000.

MURAKAMI, K.; KONDO, T.; EPSTEIN, C.J.; CHAN, P.H. Overexpression of CuZn-superoxide dismutase reduces hippocampal injury after global ischemia in transgenic mice. Stroke. v. 28, p. 1797-1804, 1997.

NAFFAH-MAZZACORATTI, M.G.; CAVALHEIRO, E.A.; FERREIRA, E.C.; ABDALLA, D.S.P.; AMADO, D.; BELLISSIMO, M.I. Superoxide dismutase, glutathione peroxidase activities and the hydroperoxide concentration are modified in the hippocampus of epileptic rats. Epilepsy Research. v. 46, p. 121-128, 2001.

SIES, H. Oxidative stress: introduction. In: Oxidative stress: oxidants and antioxidants. New York: Academic Press, p. 15-22, 1991.

SIMONIC, A.; LAGINJA, J.; VARLJEN, J.; ZUPAN, G.; ERAKOVIC, V. Lithium plus pilocarpine induced status epilepticus - biochemical changes. Neuroscience Research. v. 36, p. 157-166, 2000.

SZYNDLER, J.; BOBTOWICZ, T. W.; SKÓRZEWSKA, A.; MACIEJAK, P.; WALKOWIAK, J.; LECHOWICZ, W.; TURZYNSKA, D.; BIDZINSKI, A.; PLAZNIK, A. Behavioral, biochemical and histological studies in a model of pilocarpine-induced spontaneous recurrent seizures. Pharmacology Biochemistry and Behavior. v. 81. p. 15-23, 2005.

TURSKI, W.A.; CAVALHEIRO, E.A.; SCHWARTZ, M.; CZUCZWAR, S.J.; KLEINROK, Z.; TURSKI, L. Limbic seizures produced by pilocarpine in rats: a behavioural, electroencephalographic and neuropathological study. Behavior Brain Research. v. 9, p. 315-335, 1983.

VANHATALO, S.; RIIKONEN, R. Nitric oxide metabolites, nitrates and nitrites in the cerebrospinal fluid in children with west syndrome. Epilepsy Research. v. 46, p. 3-13, 2001.

WALZ, R.; MOREIRA, J.C.F.; BENFATO, M.S.; QUEVEDO, J.; SCHORER, N.; VIANNA, M.M.R.; KLAMT, F.; DAL-PIZZOL, F. Lipid peroxidation in hippocampus early and late after status epilepticus induced by pilocarpine of kainic acid in Wistar rats. Neuroscience Letters. v. 291, p. 179-182, 2000. 